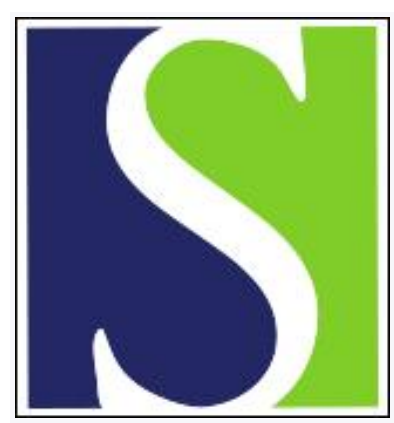

Scand J Work Environ Health 1986;12(4):362-364

https://doi.org/10.5271/sjweh.2128

Issue date: Aug 1986

Physiological response of the rat to different vibration frequencies.

by Okada $\mathrm{A}$

This article in PubMed: www.ncbi.nlm.nih.gov/pubmed/3775324

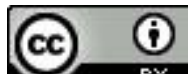




\title{
Physiological response of the rat to different vibration frequencies
}

\author{
by Akira Okada, MD, DMSc ${ }^{1}$
}

\begin{abstract}
OKADA A. Physiological response of the rat to different vibration frequencies. Scand $J$ Work Environ Health 12 (1986) 362-364. Vibration (acceleration of $50 \mathrm{~m} / \mathrm{s}^{2}$ and frequencies from 30 to $960 \mathrm{~Hz}$ ) was applied to the tail and hind legs of rats for a 4-h period. The responses of the peripheral circulatory and peripheral nervous systems, muscle function, and the central nervous system were examined. When a tail was exposed to the vibration stimulus, the skin temperature dropped significantly at 30 and $240 \mathrm{~Hz}$; the blood flow decreased significantly at 30,60 , and $480 \mathrm{~Hz}$; and the peripheral nerve conduction velocity was significantly slower at 30,60 , and $120 \mathrm{~Hz}$. After vibration of the hind legs the activity of plasma creatine phosphokinase (CPK) showed significantly high values at $30,60,120$, and $480 \mathrm{~Hz}$ and was especially high at $30 \mathrm{~Hz}$; the content of brain norepinephrine was significantly decreased at $120 \mathrm{~Hz}$ in the hypothalamus; dopamine was significantly increased at $60 \mathrm{~Hz}$ in the medulla oblongata and pons; and serotonin was significantly increased at $120 \mathrm{~Hz}$ in the hypothalamus. Thus the physiological responses of the rat to local vibration depend on, among other variables, the vibration frequency. In the case of the response of plasma CPK it would appear to depend on the amplitude (displacement) of this stimulus.
\end{abstract}

Key terms: blood flow, brain monoamines, local vibration, peripheral nerve conduction velocity, sarcoplasmic enzymes, skin temperature.

In the investigation of vibration hazards, it is important to study the effects of both the intensity of vibration (in terms of acceleration in meters per square second) and the vibration frequency (in hertz).

In the past some investigators, such as AndreevaGalanina, examined the range of vibration frequencies and related them to vibration disorders. The findings were then considered to be incidental and corresponding to the work process. In general a vibrating tool has a wide spectrum of vibration frequency. Therefore it is not easy to conclude whether or not the maximum damage to a subject can occur at or around a predominant frequency. It is important, with respect to the hand-arm vibration syndrome, to determine the frequencies which may have serious effects on the arteries, nerves, and musculoskeletal system.

A study of the relationship between vibration frequency and the observed physiological responses is best carried out on animals. In the present investigation parts of rats were exposed to constant acceleration when the frequency of the stimulus was varied. The physiological responses to each frequency were examined in the peripheral circulatory and peripheral nervous systems, in muscles (sarcoplasmic enzymes), and in the central nervous system (brain monoamines).

1 Department of Public Health, School of Medicine, Kana-
zawa University, 13-1 Takaramachi, Kanazawa 920, Japan.

Reprint requests to: Prof A Okada, Department of Public Health, School of Medicine, Kanazawa University, 13-1 Takaramachi, Kanazawa 920, Japan.

\section{Material and methods}

Male Wistar rats $(\mathrm{N}=153)$ weighing $245-302 \mathrm{~g}$ were used in the experiments. The apparatus for vibration exposure was composed of an electromagnetic shaker (Emic 513-A, shaking power $7.5 \mathrm{~kg}$; vibration frequency range 5 to $5000 \mathrm{~Hz}$ ) coupled to an amplifier (Tachikawa TA-100), a function oscillator (Trio AG202A), and a vibration meter (Emic 505-D).

The animals were individually placed in wire mesh cages, in the prone position. Vertical and sinusoidal vibration at different frequencies $(30,60,120,240,480$, $960 \mathrm{~Hz}$ ) but under constant acceleration $\left(50 \mathrm{~m} / \mathrm{s}^{2}\right)$ were applied to the tail and hind legs of the rats for $4 \mathrm{~h}$.

In experiment $A$ each vibration frequency was applied to the tail of the rats for investigating the response of the peripheral circulatory and nervous systems. The tail was outside the cage and was horizontally fixed on the vibrating plate. The cage containing the body of the rat was fixed on a nonvibrating plate separated from the shaker. The effects were observed through measurements of the skin temperature of the tail and the blood flow and peripheral nerve conduction velocities on the tail before and after vibration exposure. Blood flow was measured by the hydrogen gas clearance method using electrolysis $(1,4)$. The differences between the mean values before and after the exposure were examined by Student's paired t-test.

In experiment $B$ each vibration frequency was applied to the hind legs of rats. The hind legs were outside the cage, and the plantar surfaces were horizontally fixed on the vibrating plate by means of double-sided adhesive tape so that the vibration would be transmitted only to the hind legs. The cage was fixed on a non- 
vibrating plate separated from the shaker. The activities of plasma sarcoplasmic enzymes, creatine phosphokinase, aldolase, and lactate dehydrogenase, were examined in the investigation of the effect on muscle function. Soon after the vibration exposure, arterial blood was drawn from the abdominal aorta by means of a heparinized syringe under anesthesia. Activities of plasma total creatine phosphokinase and its isoenzyme, the muscle fraction of creatine phosphokinase, plasma aldolase, and plasma lactate dehydrogenase were determined $(5,6)$.

In experiment $C$ each vibration was applied to the hind legs of rats. Brain monoamines, norepinephrine (NE), dopamine (DA), and serotonin (5-HT) were examined to investigate the effect on the central nervous system. The rats were decapitated immediately after the vibration exposure. The brain was quickly removed from the cranium. For regional studies, the brain was divided into seven regions (2). The levels of these amines were determined fluorometrically (3).

In experiments $B$ and $C$, the control rats were placed near the operating shaker under the same conditions ( $4 \mathrm{~h}$ in wire mesh cages and in the prone position).

Student's t-test was used to test the significance of the differences between the mean values of the vibration-exposed and control rats.

\section{Results}

Figures 1, 2, and 3 show the effects on the tail exposed to $4 \mathrm{~h}$ of local vibration at the different frequencies under the constant acceleration of $50 \mathrm{~m} / \mathrm{s}^{2}$. The skin temperature after the vibration exposure was significantly lower than before the exposure at 30 and $240 \mathrm{~Hz}$, and the blood flow after the vibration exposure was significantly less than before it at 30,60 , and $480 \mathrm{~Hz}$. The peripheral nerve conduction velocity after the vibration exposure was significantly slower than before it at 30,60, and $120 \mathrm{~Hz}$.

Figure 4 shows the values of the total and muscle fraction of plasma creatine phosphokinase for each vibration frequency. Compared with those of the control group, each value of the exposed group was significantly higher after exposure to the frequencies between 30 to $480 \mathrm{~Hz}$. The value for $960 \mathrm{~Hz}$ showed no significant difference between the exposed and the control groups. The activities of both the plasma aldolase and lactate dehydrogenase were not significantly affected by any vibration frequency.

Of the responses of norepinephrine, dopamine, and serotonin in the whole brain to each vibration frequency, on the other hand, norepinephrine showed a tendency to decrease after local vibration exposure at $120 \mathrm{~Hz}$; dopamine and serotonin showed no particular change at any frequency.

Figure 5 shows the responses of three amines in the various regions of the brain. Norepinephrine showed a tendency to decrease at $60 \mathrm{~Hz}$ and decreased significantly at $120 \mathrm{~Hz}$ in the hypothalamus. Norepinephrine

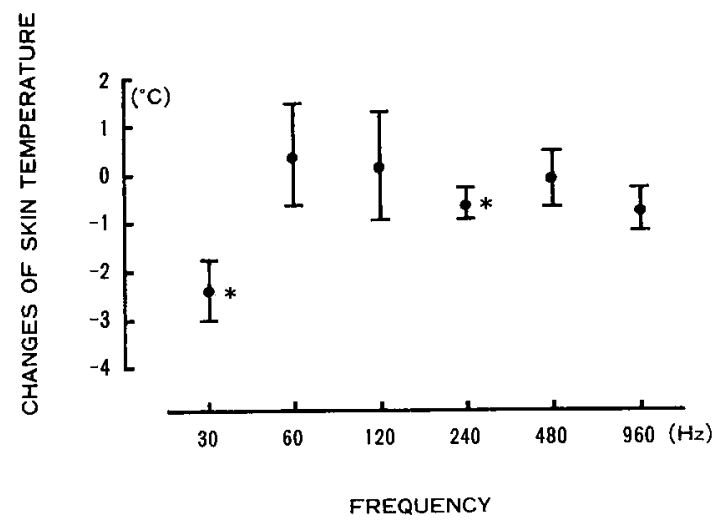

Figure 1. Effect of various vibration frequencies on the skin temperature of the tails of rats exposed to $4 \mathrm{~h}$ of local vibration under a constant acceleration $\left(50 \mathrm{~m} / \mathrm{s}^{2}\right)$. Each group represents the mean and standard error of the mean of the change in the skin temperatures of four rats at 60,240 , and $960 \mathrm{~Hz}$ or five rats at 30,120 , and $480 \mathrm{~Hz}$ [ ${ }^{*} \mathrm{p}<0.05$ (t-test)].

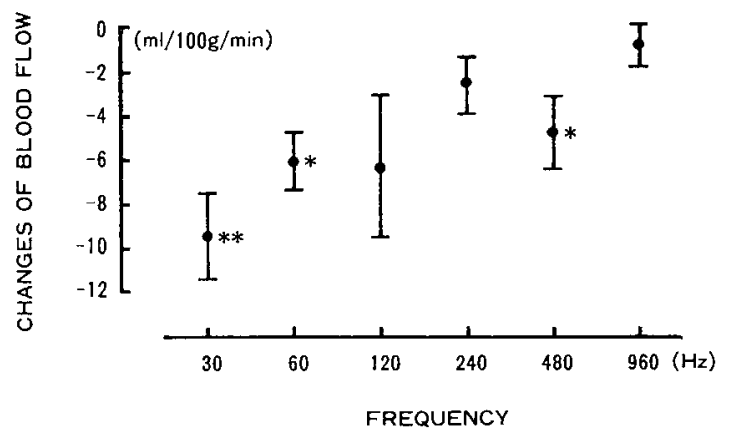

Figure 2. Effect of various frequencies on blood flow in the talls of rats exposed to $4 \mathrm{~h}$ of local vibration under a constant acceleration $\left(50 \mathrm{~m} / \mathrm{s}^{2}\right)$. Each group represents the mean and standard error of the mean of the change in blood flow of four rats at 60,240 , and $960 \mathrm{~Hz}$ or five rats at 30,120 , and $480 \mathrm{~Hz}$ $\left[{ }^{*} p<0.05,{ }^{* \star} p<0.01\right.$ (t-test)].

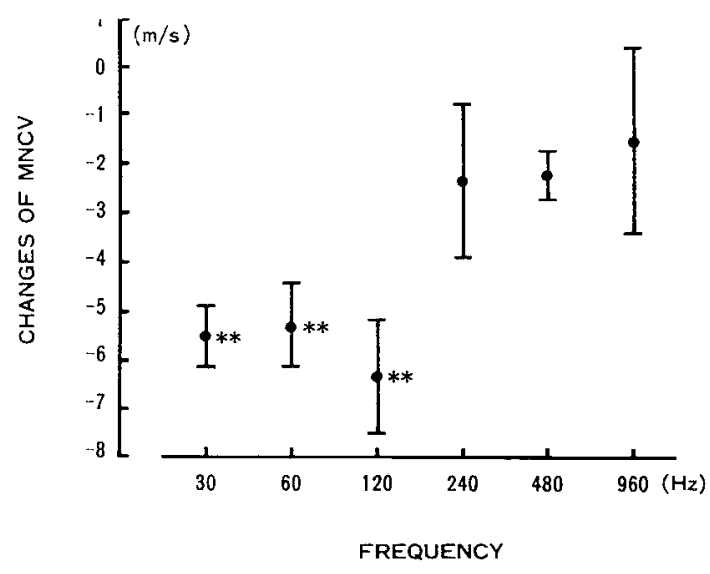

Figure 3. Effect of various frequencies on peripheral motor nerve conduction velocity (MNCV) in the tails of rats exposed to $4 \mathrm{~h}$ of local vibration under a constant acceleration $\left(50 \mathrm{~m} / \mathrm{s}^{2}\right)$. Each group represents the mean and standard error of the mean of the change in MNCV of four rats at 60,240 , and $960 \mathrm{~Hz}$ or five rats at 30,120 , and $480 \mathrm{~Hz}\left[^{* \star} \mathrm{p}<0.01\right.$ (t-test)]. 


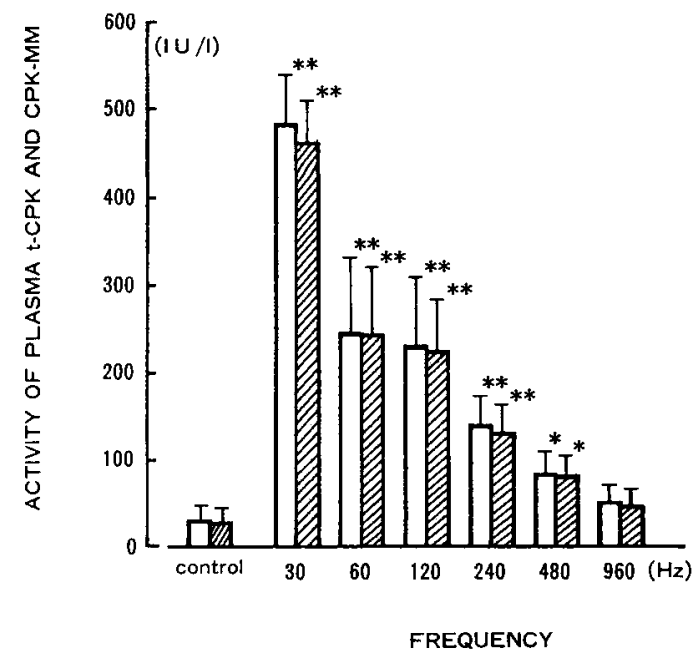

Figure 4. Effects of various frequencies on the activities of the total creatine phosphokinase (t-CPK) and the muscle fraction of creatine phosphokinase (CPK-MM). The hind legs of the rats were exposed to local vibration under a constant acceleration $\left(50 \mathrm{~m} / \mathrm{s}^{2}\right)$ for $4 \mathrm{~h}$. Each group represents the mean and standard deviation for five rats $\left[{ }^{*} \mathrm{p}<0.01\right.$, ${ }^{*} \mathrm{p}<0.001$ (t-test)]. ( $[$ ] $\mathrm{t}$-CPK, DRA CPK-MM).

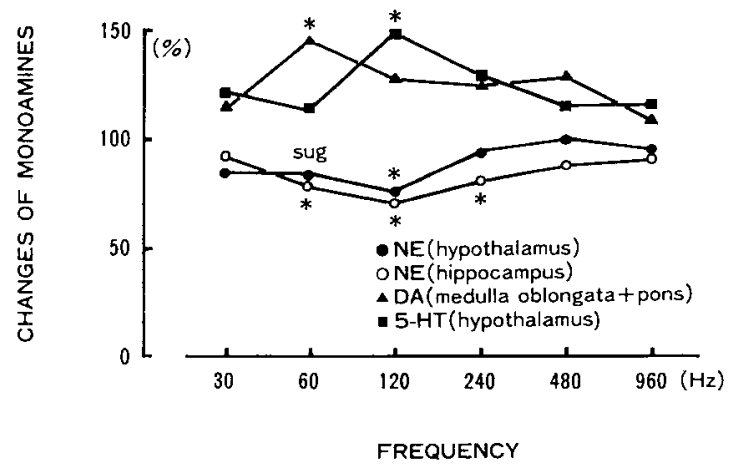

Figure 5. Effect of various frequencies on the levels of norepinephrine (NE), dopamine (DA), and serotonin (5-HT) in the affected brain regions. The hind legs of the rats were exposed to local vibration under a constant acceleration $\left(50 \mathrm{~m} / \mathrm{s}^{2}\right)$ for $4 \mathrm{~h}$. The ratios of the mean content of four rats exposed to vibration to that of four control rats are illustrated [sug $\mathrm{p}<$ $0.10, * p<0.05$ (t-test)].

decreased significantly at 60,120 , and $240 \mathrm{~Hz}$ in the hippocampus. Dopamine showed a significant increase at $60 \mathrm{~Hz}$ in the medulla oblongata and pons. Serotonin showed a significant increase at $120 \mathrm{~Hz}$ in the hypothalamus. In the other regions of the brain, the amines were not notably affected.

\section{Discussion}

From the present study the following conclusions can be drawn. The peripheral nervous system of rats was affected by low frequencies from 30 to $120 \mathrm{~Hz}$, whereas the peripheral circulatory system was influenced not only by low frequencies ( 30 and $60 \mathrm{~Hz}$ ) but also by high frequencies such as 240 and $480 \mathrm{hz}$.

The response of plasma creatine phosphokinase activity was related to the effect of vibration on muscle function, and the increase in the total plasma creatine phosphokinase activity was caused by the increase in the muscle fraction of the plasma creatine phosphokinase, which originated in the skeletal muscle. The activity showed the highest value, in comparison with other vibration frequencies, at $30 \mathrm{~Hz}$. The rate of increase in plasma creatine phosphokinase activity showed a tendency to decrease gradually with the increase in vibration frequency. Therefore, the response of plasma creatine phosphokinase seems to depend on the amplitude of displacement. In the present experiment, pathohistological observation of the hind legs of the rats exposed to vibration was also performed. There were no findings of structural changes suggesting any muscle tissue destruction. Therefore, it is presumed that the increase in the plasma creatine phosphokinase activity induced by local vibration is attributable to the rise of permeability in the muscle-cell membrane.

There were no meaningful changes in the three monoamines (norepinephrine, dopamine, and serotonin) determined in the whole brain. On the other hand, in some regions of the brain, each amine was affected differently. These changes were observed at frequencies of 60,120 , and $240 \mathrm{~Hz}$. The affected regions were the medulla oblongata and pons, the hypothalamus, and the hippocampus. The degree of change, however, was small. Therefore the effect of local vibration on the central nervous system appears to be relatively small.

Thus the physiological responses of rat to local vibration depend on the vibration frequency. In the case of plasma creatine phosphokinase, the response seems to depend on the amplitude of displacement.

\section{References}

1. Aukland K, Bower BF, Berliner RW. Measurement of local blood flow with hydrogen gas. Circ Res 14 (1964) 164-187.

2. Glowinski J, Iversen IL. Regional studies of catecholamines in the rat brain - I. J Neurochem 13 (1966) $655-669$.

3. Karasawa T, Fukuda K, Yoshida K, Shimizu M. A double column procedure for simultaneous estimation of norepinephrine, normetanephrine, dopamine, 3-methoxytyramine and 5-hydroxytryptamine in brain tissues. Jpn J Pharmacol 25 (1975) 727-736.

4. Stosseck K, Luebbers DW, Cottin N. Determination of local blood flow (microflow) by electrochemically generated hydrogen: Construction and application of the measuring probe. Pfluegers Arch 348 (1974) 225-238.

5. Takahashi K, Uchikubo S, Oimomi H, Shinko T. Creatine phosphokinase isoenzymes of human heart muscle and skeletal muscle. Clin Chim Acta 38 (1970) 285-290.

6. Wroblewski FF, La Due JS. Lactic dehydrogenase activity in blood. Proc Soc Exp Biol Med 90 (1955) 210-213. 